Proc. Indian Acad. Sci. (Chem. Sci.), Vol. 92, Numbers 4 \& 5, August \& October 1983, pp. 449-456. (C) Printed in India.

\title{
Crystallographic study of push-pull ethylenes
}

\author{
D ADHIKESAVALU, NIRUPA U KAMATH and K VENKATESAN \\ Department of Organic Chemistry, Indian Institute of Science, Bangalore 560012, India
}

\begin{abstract}
From structural data of several crystal structure analyses of push-pull ethylenes it is found that $\mathrm{C}=\mathrm{C}$ bond lengths in this class of molecules are significantly longer than the value for this bond in ethylene. With powerful donors and acceptors such as $-\mathrm{NMe}_{2}$ and $-\mathrm{COOMe}$ groups respectively, the $\mathrm{C}=\mathrm{C}$ bond length is as long as $1.460 \mathrm{~A}$. Correlations are observed between (i) $\mathrm{C}=\mathrm{C}$ bond lengths and the twist angles about the $\mathrm{C}=\mathrm{C}$ bond and (ii) $\mathrm{C}=\mathrm{C}$ bond lengths and the torsional barrier measured by the DNMR technique.
\end{abstract}

Keywords. $\mathrm{X}$-ray structure analysis; push-pull ethylenes; $\mathrm{C}=\mathrm{C}$ bond lengths; rotational barriers.

\section{Introduction}

Ethylene substituted with electron donating and electron accepting groups in the vicinal positions is known as polarized or push-pull ethylenes.

The conjugative interaction between the donor and acceptor parts of the molecule through the ethylene $\pi$ bond results in interesting changes in chemical and physical properties of this class of compounds. For example, in 1-dimethyl amino-2-acetyl ethylene (figure 1) the conjugation gives rise to resonating structures (I) and (II). The $\mathrm{C}=\mathrm{C}$ bond in such systems are resistant to oxidation and reduction and to the addition of bromine under normal conditions (Jensen and Henricksen 1968). Whereas the barrier to rotation about the $\mathrm{C}=\mathrm{C}$ bond in 1,2-dideuterioethylene is $62 \mathrm{kcal} \mathrm{mol}^{-1}$, it is substantially reduced in polarized ethylenes (Sandström and Wennerbeck 1971; Shvo and Shanan-Atidi 1969).

There are two important factors which determine the conformation about the ethylene bond in this class of molecules: (i) intramolecular steric interaction between the substituents on the double bond, and (ii) loss of $\pi$-electron energy $\left(E_{\pi}\right)$ as the rotation about the $\mathrm{C}=\mathrm{C}$ bond increases.

The conjugation is maximum in the planar state and as such this will favour a planar geometry. The steric interaction between the donor and acceptor groups is minimum at the dihedral angle of $90^{\circ}$ and so, a $90^{\circ}$ twisted conformation will be favoured.

Push-pull ethylenes can be represented by the general formula I as shown below

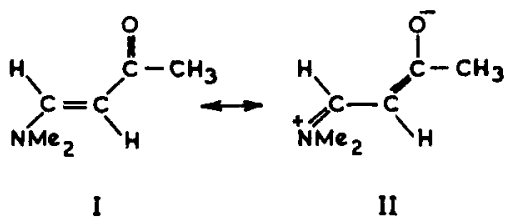

Figure 1. Resonance structures of 1-dimethyl amino-2-acetyl ethylene. 
where $D_{1}$ and $D_{2}$ represent electron-donating groups and $A_{1}$ and $A_{2}$ electron accepting groups.

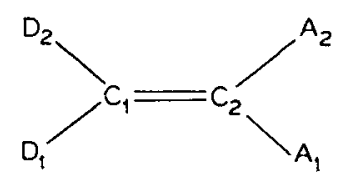

The twist about the $\mathrm{C}=\mathrm{C}$ double bond is measured as the angle between the plane through the atoms $D(1), D(2)$ and $C(1)$ and the plane through the atoms $A(1), A(2)$ and $C(2)$.

Although results based on NMR and other techniques have been analysed in literature (Sandström 1983), no such detailed discussion of the x-ray results has been reported. In recent years, we have been investigating the molecular structures of a large number of push-pull ethylenes via $\mathrm{X}$-ray crystallography and in this paper we discuss our findings.

\section{Results and discussion}

In table 1 are recorded the bond lengths of the ethylene bond and the twist angles for the push-pull ethylenes investigated in our laboratory (Adhikesavalu and Venkatesan 1981, 1982, 1983a, b; Nirupa Kamath and Venkatesan 1983) and those by Abrahamsson et al (1974). The present results of the push-pull ethylenes are based on the intensity data collected on a Nonius CAD-4 diffractometer and the final $R$-factors are in the range $4-5.8 \%$ with the exception of 14 in table 1 for which the $R$-value at the present stage of refinement is around $10 \%$. In most of the cases investigated, the samples were kindly provided by Professor J. Sandström. Also recorded in this table are the values of the rotational barrier about the $\mathrm{C}=\mathrm{C}$ bond measured using the DNMR technique (Sandström 1983). It may be noted that in molecules 1 to 4 (table 1), whereas the donor groups differ, the acceptor groups remain the same.

Comparing 1 and 2 , on the basis of electron donating capacity, the presence of two dimethyl amino groups in 1 would be expected to lead to a longer $\mathrm{C}=\mathrm{C}$ length than in 2 in which the donor groups are-SMe and-NMe groups. But this is not borne out by the $\mathrm{x}$-ray results. The structural evidence shows that, due to steric interaction the $\mathrm{N}$-methyl groups are twisted by $33^{\circ}$ in 1 whereas in 2 it is of lower magnitude $\left(23^{\circ}\right)$ with respect to the plane defined by the two donor atoms and the olefinic carbon atoms. The twist about the $\mathrm{C}=\mathrm{C}$ bond is $29.3^{\circ}$ in $\underline{1}$ and $21.7^{\circ}$ in 2 . Comparing the molecule $\underline{1}$ having an acyclic donor with $\underline{3}$ containing a cyclic donor, we observe that the $\mathrm{C}=\mathrm{C}$ bond length is, within experimental error, same although there is a difference of about $9^{\circ}$ in the twist angle. Cyclisation in 3 and 14 has reduced the steric interaction between the donor and acceptor parts of the molecule. Similarly, the larger twist in $4\left(31.5^{\circ}\right)$ compared to that in $\underline{3}\left(20.2^{\circ}\right)$ and $14\left(22.9^{\circ}\right)$ is due to the greater steric interaction in the planar state in 4 . The average value of the exocyclic angle (i.e. $C_{2}-C_{1}-D_{1}$ and $C_{2}-C_{1}-D_{2}$ ) in 4 is significantly smaller $\left(120.6(5)^{\circ}\right)$ than in $\underline{3}\left(124.8(2)^{\circ}\right)$. However, the significantly longer $\mathrm{C}=\mathrm{C}$ bond length in $4(1.429(6) \AA)$ compared to that in $3(1.407(3) \AA)$ cannot be due to the difference of about $11^{\circ}$ in twist angles. In this connection it is noteworthy that the difference in the twist angles between 5 and 7 is about $16^{\circ}$ (table 1 ) but there is no significant difference in the $\mathrm{C}=\mathrm{C}$ bond length. These observations seem to indicate that the hexahydropyrimidine ring possesses better electron donating ability than the five-membered imidazolidine ring. 
Table 1.

\begin{tabular}{|c|c|c|c|c|}
\hline $\begin{array}{l}\text { SI. No. A } \\
\text { Reference } \\
\text { code }\end{array}$ & structural tormula & $\begin{array}{c}\text { Rotational } \\
\text { barrier } \\
\text { about the } \\
c=c \text { bond } \\
\text { ik cals mole- }\end{array}$ & $\begin{array}{l}\text { Twist about } \\
\text { the } c=\frac{6}{101} \\
\text { bond }\end{array}$ & $\begin{array}{l}c=c \text { bond } \\
\text { length }(\lambda)\end{array}$ \\
\hline 1 & & 一 & 29.3 & $1.407(4)$ \\
\hline 2 & & 13.2 & 21.7 & $1.397(8)$ \\
\hline 3 & & - & 20.2 & $1.407(3)$ \\
\hline 4 & & - & 31.5 & $1.429(6)$ \\
\hline 5 & & $<8$ & 56.8 & $1.461(2)$ \\
\hline 6 & & 一 & 5.0 & $1.451(3)$ \\
\hline 7 & & $<8$ & 72.9 & $1.468(3)$ \\
\hline 8 & & $<8$ & 62.6 & $1.684(2)$ \\
\hline 9 & & 一 & 38.3 & $1.416(6)$ \\
\hline 10 & & 9.5 & 41.0 & $1.448(4)$ \\
\hline 11 & & 19.0 & 15.0 & $1.369(7)$ \\
\hline 17 & & 19.1 & 12.8 & $1.377(3)$ \\
\hline 13 & & 9.8 & 34.8 & $1.409(4)$ \\
\hline 14 & & 9.5 & 22.9 & $1.415(8)$ \\
\hline
\end{tabular}

E.S.d's are given in parenthesis. References: X-ray work on 1-4, 6-9 (Adhikesavalu and Venkatesan); 5, 12-14 (Nirupa Kamath and Venkatesan). Rotational barriers on 5, 7, 8, 13 (Wennerbeck and Sandström 1972); 10, 11 (Abrahamsson et al 1974); 12 (Dreier et al $\overline{1} \overrightarrow{9} \overline{8}$ ); $\overline{14}$ (Sandström et al 1977); 2 (Sandström and Wennerbeck 1978). 
The barrier to rotation in 1 could not be measured due to symmetry reasons (Kessler 1968). Also, the barriers to rotation for 3 and 4 have not been reported. But, for similar systems the barrier is reported to be less than $10 \mathrm{kcals} / \mathrm{mol}$ (Sandström and Wennerbeck 1971). However, NMR studies have shown that the energy barrier about the $\mathrm{C}=\mathrm{C}$ bond is less in molecules with electron donating groups present as part of a ring than in those with acyclic systems. The barrier to rotation in 2 seems to indicate that the transition state is stabilized better in this molecule compared to 1 .

The combined effect of the powerful electron donating - $\mathrm{NMe}_{2}$ group and the strong acceptor combination of acetyl and carbomethoxy groups in $\underline{5}$ to $\underline{8}$ produce a remarkable lengthening of the $\mathrm{C}=\mathrm{C}$ bond $(1.460(3) \AA)$. The slightly smaller value of $\mathrm{C}=\mathrm{C}$ bond length in $\underline{6}(1.451(3) \mathrm{A})$ compared to the values obtained for $\underline{5}, \underline{7}$ and $\underline{8}$ may be attributed to the considerably smaller angle of twist in 6 . It is pertinent to observe that the theoretical calculations by MINDO/2 method (Dewar and Haselbach 1970) have shown that the $\mathrm{C}=\mathrm{C}$ bond length extension in ethylene, on twisting about the $\mathrm{C}=\mathrm{C}$ bond by $90^{\circ}$ is only $0.03 \AA$. The $\mathrm{C}=\mathrm{C}$ bond length calculated for $90^{\circ}$ twisted state is 1.367 A compared to the normal value of 1.336(2) A (Kuchitsu 1966).

There would be severe steric interaction involving $-\mathrm{NMe}_{2}$ groups at the donor end and the acetyl groups at the acceptor end in the planar state in 7 and 8 . To relieve this strain, the molecules are twisted $72.9^{\circ}$ in $\underline{7}$ and $62.6^{\circ}$ in $\underline{8}$ about the $\mathrm{C}=\mathrm{C}$ bond. The carbomethoxy group introduces less steric strain compared to an acetyl group and the lesser twist in 8 may be due to this. In the absence of the $-\mathrm{NMe}_{2}$ group, we should expect the twist angle to be less, but the unexpectedly low value of $5^{\circ}$ in molecule 6 is attributed to the two intramolecular $\mathrm{N}-\mathrm{H}$. . . O bonds (figure 2). In fact the twist angle corresponding to the minimum energy conformation, calculated using semiempirical methods (Ramachandran and Sasisekharan 1968), is $22^{\circ}$. However, when the contribution of the hydrogen bonding energy is introduced in the conformational energy calculations, the conformation with a twist angle of $8^{\circ}$ is favoured.

${ }^{1} \mathrm{H}$ NMR spectra of 7 under conditions of slow exchange shows two forms, one with diastereotopic and the other with homotopic-COMe protons. The first must be

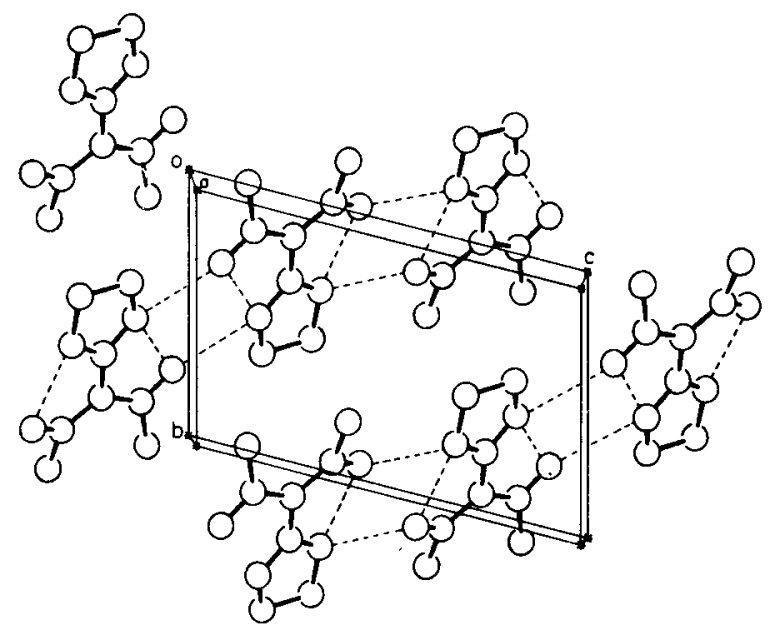

Figure 2. Intramolecular $\mathrm{N}-\mathrm{H} \ldots \mathrm{O}$ bonding in 6 . 
degenerate $E Z-Z E$ pair, whereas the second may be $E E$ or $Z Z$ (figure 3). But the $E E$ form is less likely because of the strong repulsion to be expected between parallel dipoles as shown by the effect of solvent polarity and in particular by the ASIS (Sandström 1983).

In the solid state, 6 adopts the $Z Z$ conformation favoured by the intramolecular hydrogen bonding. In $\underline{5}, \underline{7}$ and $\underline{8}$ the acceptor groups assume $E Z$ conformation.

The alkylthio groups have been found to be poor donors and this is clearly seen in the shorter $C=C$ bond length $(1.369(7) A)$ in 11 . Comparison of the $C=C$ lengths obtained for 9 and 11 , shows again that the thio group is a poor donor. As expected then, the ketene mercaptals being weak donors, the bond length in 12 is $1.377(3) \mathrm{A}$. In both cases the rotational barrier has been measured and is of the order of $19 \mathrm{kcals} \mathrm{mol}^{-1}$.

The combination of an acetyl and phenyl group in 13 as acceptor groups does not seem to produce the same degree of delocalization as the acetyl-carbomethoxy combination does as, for example, observed in $\underline{5}$ with the same donor substituents in both the molecules.

\section{Conclusions}

From the present $x$-ray crystallographic results the following significant conclusions may be drawn:

(i) The larger $\mathrm{C}=\mathrm{C}$ bond length observed in molecules 5 to 8 compared to those of 1 to 4 clearly shows that the -COMe, -COMe or -COMe, -COOMe substituents as acceptors are definitely better than the $-\mathrm{CN}$ group as acceptors. Further, as noted

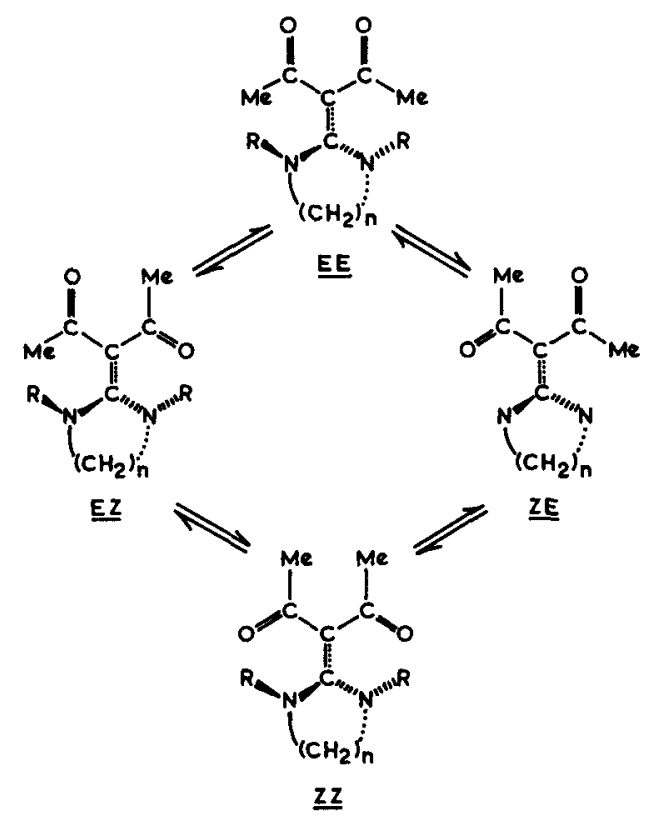

Figure 3. Conformational possibilities of acetyl group. 
earlier, the hexahydropyrimidine ring appears to be a better donor than the imidazolidine ring.

(ii) In figure 4, the $\mathrm{C}=\mathrm{C}$ bond lengths are plotted against the observed twist angle about the $\mathrm{C}=\mathrm{C}$ bond. It is observed that the nature of the correlation for the molecules in which the donors are acyclic is different from that for those with cyclic donors. For the same angle of rotation, the $\mathrm{C}=\mathrm{C}$ bond length tends to be longer in the molecules with cyclic donors than the ones with acyclic donors. However, for angles of rotation above $55^{\circ}$, the $\mathrm{C}=\mathrm{C}$ bond lengths tend to be very nearly equal in both the cases. The deviation of molecule 6 from the curve (b) arises from the intramolecular $\mathrm{N}-\mathrm{H}$. . O O hydrogen bonding in this molecule. The observed difference depicted in figure 4 with acyclic (a) and cyclic (b) donors could be partly due to the difference in steric factors between these two classes of molecules although it is known from theoretical calculations (Dewar and Haselbach 1970) that the variation expected in $\mathrm{C}=\mathrm{C}$ bond length from the twist about this bond is less than $0.03 \mathrm{~A}$. Thus the importance of the possible difference in the electronic factor between the cyclic and acyclic donors cannot be overruled.

(iii) It is reasonable to expect a correlation between $d\left(\mathrm{C}_{s p}{ }^{2-\mathrm{N}}\right)$ and $d(\mathrm{C}=\mathrm{C})$. However, it is found from the scatter plot of $\mathrm{C}=\mathrm{C}$ and $\mathrm{C}\left(s p^{2}\right)-\mathrm{N}$, that there is a correlation corresponding to cyclic donors molecules but not when the donors are acyclic (figure 5). In the molecules with acyclic donors such as $-\mathrm{NMe}_{2}$ and $-\mathrm{SMe}$, there is rotation about $\mathrm{N}-\mathrm{Me}$ and $\mathrm{S}-\mathrm{Me}$ bonds in order to overcome the steric interaction between the donor and acceptor groups. However, this is not possible to the same degree on the molecules with cyclic donors. This provides an explanation for the absence of correlation in molecules with acyclic donors.

(iv) It was pointed out earlier that the rotational barrier about the $\mathrm{C}=\mathrm{C}$ bond in

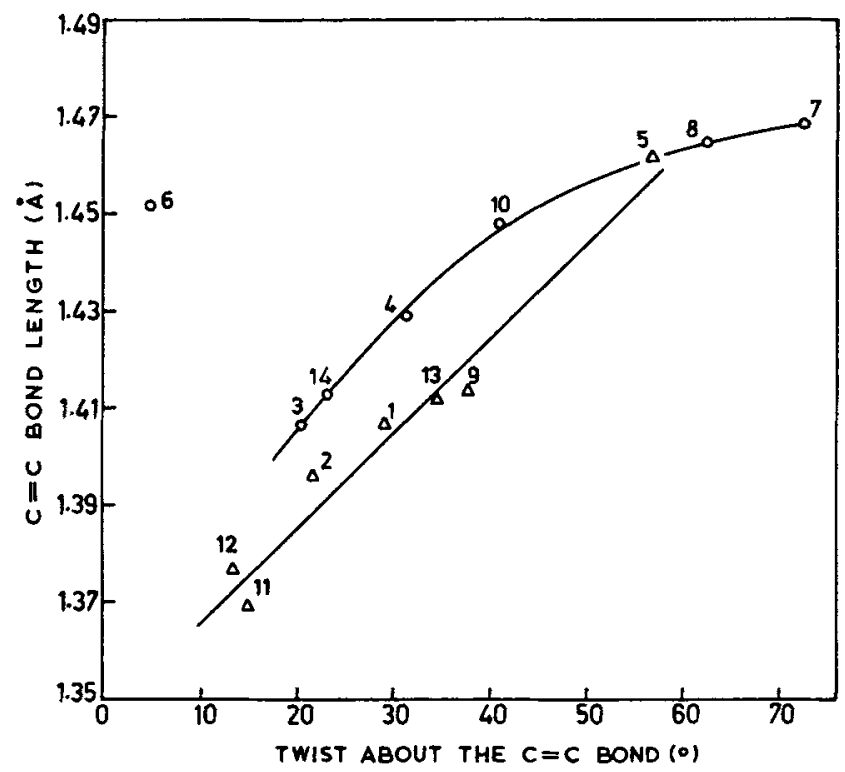

Figure 4. Plot of $\mathrm{C}=\mathrm{C}$ bond length (A) vs twist about the $\mathrm{C}=\mathrm{C}$ bond $\left({ }^{\circ}\right) . \Delta$ acyclic donors and 0 cyclic donors. 


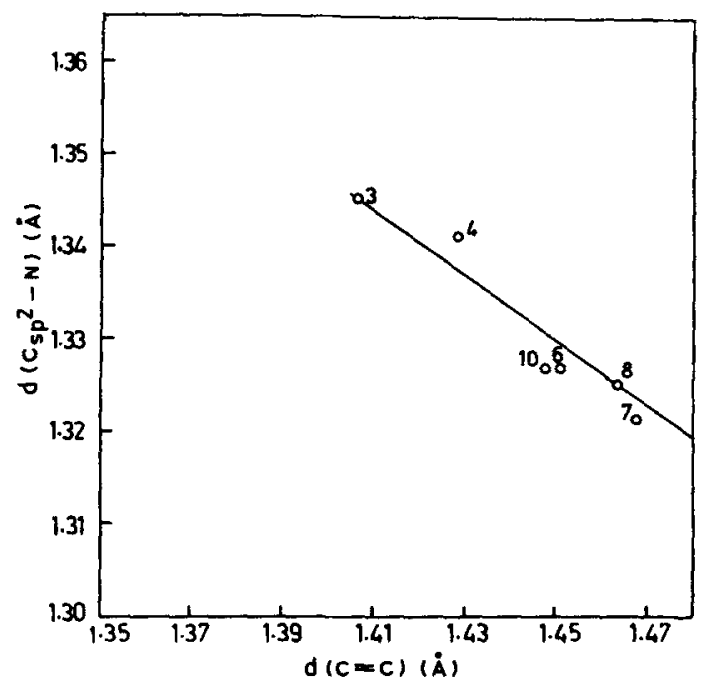

Figure 5. Correlation plot of $d\left(\mathrm{C}_{s p^{2}}-\mathrm{N}\right)$ us $d(\mathrm{C}=\mathrm{C})(\mathrm{A})$.

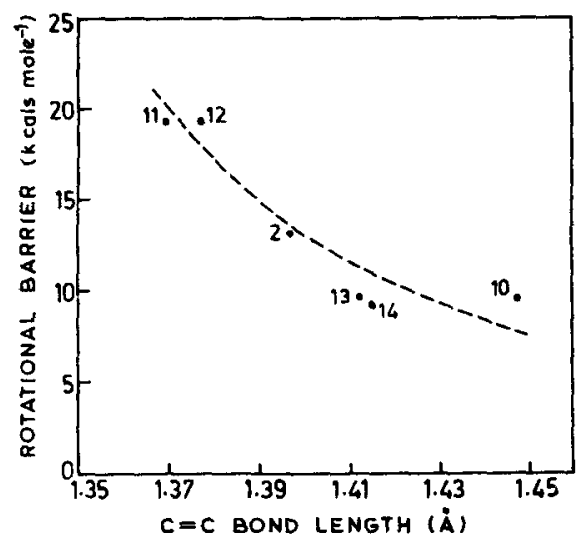

Figure 6. Plot of rotational barrier ( $\mathrm{kcal} \mathrm{mol}^{-1}$ ) vs $\mathrm{C}=\mathrm{C}$ bond length (A).

polarized ethylenes is substantially reduced. From the $\mathrm{x}$-ray results reported here it is seen that the $\mathrm{C}=\mathrm{C}$ bond length can be as large as $1.460 \mathrm{~A}$. The rotational barrier value may be expected to decrease as the $\mathrm{C}=\mathrm{C}$ bond length increases. The rotational barrier values for quite a few cases are not available from the NMR data either because the low torsional barriers of $\mathrm{C}=\mathrm{C}$ double bond are sometimes below the limit for DNMR technique ca $5 \mathrm{kcal} \mathrm{mole}^{-1}$ for these type of compounds or due to experimental difficulties.

In figure 6 are plotted $\mathrm{C}=\mathrm{C}$ bond lengths $v s$ the rotational barriers. The donor and acceptor groups being different in these molecules, the intramolecular steric strains will be different. However, there is a remarkable correlation between $\mathrm{C}=\mathrm{C}$ bond length and rotational barrier. 
(v) If we make the reasonable assumption that the electronic effects are more important in lengthening the $\mathrm{C}=\mathrm{C}$ bond, then from the crystallographic data recorded in table 1 for molecules $\underline{3}, \underline{6}, \underline{7}, \underline{8}$ and $\underline{10}$ we arrive at the conclusion that the electron withdrawing power of groups are in the order

$$
-\mathrm{COMe} \approx-\mathrm{COOMe}>\mathrm{Ph}-\mathrm{C}=\mathrm{O}>\mathrm{CN} \text {. }
$$

(vi) It has been shown from a feasibility study (Trueblood and Dunitz 1983) that from the internal molecular motions in crystals an estimation of force constants, frequencies and barriers from diffraction data can be obtained. The rotational barriers from NMR measurements are available (Sandström 1983) for many of the polarized ethylenes and the $x$-ray data are based on diffractometric measurements. It was, therefore, tempting to use the thermal motion parameters for the calculation of the rotational barriers. From the point of view of Hirshfeld's (1976) 'rigid-bond' test only three cases $(2, \underline{5}, 13$ in table 1) appeared reasonable for the estimation of rotational barriers. But it was very disconcerting to note that the rotational barrier values obtained even for these were widely different from those obtained from the NMR measurements. Although crystal environment may be responsible to some degree for the discrepancy it is clear that far more accurate intensity data than we have now are required to recover the pin from the haystack.

\title{
Acknowledgements
}

The authors thank the csiR and the UGC for financial support. They are grateful to Prof. J Sandström, for many valuable comments.

\section{References}

\author{
Abrahamsson S, Rehnberg G, Liljefors T and Sandström J 1974 Acta Chem. Scand. B28 1109 \\ Adhikesavalu D and Venkatesan K 1981 Acta Crystallogr. B37 2048 \\ Adhikesavalu D and Venkatesan K 1982 Acta Crystallogr. B38 855 \\ Adhikesavalu D and Venkatesan K 1983a Acta Crystallogr. C39 589 \\ Adhikesavalu D and Venkatesan K 1983b Acta Crystallogr. (in press) \\ Dewar J S and Haselbach E $1970 \mathrm{~J}$. Am. Chem. Soc. 92590 \\ Dreier C, Henricksen L, Karlsson S and Sandström J 1978 Acta Chem. Scand. 832281 \\ Hirshfeld F L 1976 Acta Crystallogr. A32 239 \\ Jensen K A and Henricksen L 1968 Acta Chem. Scand. 221107 \\ Kessler H 1968 Angew. Chem. Inter. Edit. 7, No. 11898 \\ Kuchitsu K 1966 J. Chem. Phys. 44906 \\ Nirupa Kamath U and Venkatesan K 1983 (Unpublished results) \\ Ramachandran C N and Sasisekharan V 1968 Advances in protein chemistry (eds) C B Anfinson Jr., M L \\ Anson, J T Edsall and F M Richards 23283 (New York: Academic Press) \\ Sandström J and Wennerbeck I 1971 Chem. Commun. 1088 \\ Sandström J, Sjostrand U and Wennerbeck I 1977 J. Am. Chem. Soc. 994526 \\ Sandström J and Wennerbeck I 1978 Acta Chem. Scand. B32 421 \\ Sandström J 1983 in Topics in stereochemistry, (eds.) L Norman, Allinger, Ernest L Eliel and Samuel H Wilen \\ Vol 14 \\ Shvo Y and Shanan-Atidi H $1969 \mathrm{~J}$. Am. Chem. Soc. 916683 \\ Trueblood K N and Dunitz J D 1983 Acta Crystallogr. B29 120 \\ Wennerbeck I and Sandström J 1972 J. Org. Magn. Reson. 4783
}

